\title{
Splenic Gestation: A rare case report
}

\section{Narendra R. Patil*, Shivaji D. Birare, Apurva R. Dale, Sanjay K. Mahule}

Department of Pathology, Government Medical College and Hospital, Latur, Maharashtra, India

Received: 09 April 2017

Accepted: 02 May 2017

\section{*Correspondence:}

Dr. Narendra R. Patil,

E-mail: mdslatur@gmail.com

Copyright: (c) the author(s), publisher and licensee Medip Academy. This is an open-access article distributed under the terms of the Creative Commons Attribution Non-Commercial License, which permits unrestricted non-commercial use, distribution, and reproduction in any medium, provided the original work is properly cited.

\begin{abstract}
Abdominal ectopic pregnancy accounts for only $1.3 \%$ of all ectopic pregnancies and occurs when a fertilised ovum implants in an extra pelvic peritoneal location. Splenic pregnancy is one of the least common form of abdominal ectopic pregnancy. Early diagnosis is essential as delay in treatment carries significant potential for morbidity and mortality. We present a case of 22-year-old female with 6 weeks amenorrhoea and acute abdomen. Patient underwent emergency exploratory laparotomy with suspicion of ?splenic hemangioma ?ruptured ectopic pregnancy. Splenectomy was performed. Final diagnosis was confirmed on histopathology showing chorionic villi invading the splenic parenchyma.
\end{abstract}

Keywords: Beta human chorionic gonadotropin, Computerized tomography scan, In-vitro fertilization, Magnetic resonance imaging, Ultrasonography

\section{INTRODUCTION}

Extrauterine or ectopic pregnancy occurs when the fertilized ovum becomes implanted in tissue other than the endometrium. ${ }^{1}$ Ectopic pregnancies account for $2 \%$ of all pregnancies and $9 \%$ of all pregnancy related deaths. ${ }^{2}$ Ectopic pregnancy remains a leading cause of maternal deaths in early pregnancy. The most common site of ectopic implantation is within the fallopian tube, accounting for approximately $95.5 \%$ of all ectopic gestations. $^{3}$

Although rare, extra tubal pregnancies represent some of the most serious complications of pregnancy. Approximately $1.3 \%$ of ectopic pregnancies are abdominal. ${ }^{3}$ Primary abdominal pregnancies have been described in a variety of extra pelvic organs including omentum, liver and small and large intestine. ${ }^{4}$ The spleen is one of the rarest sites for primary abdominal pregnancy. Preoperative diagnosis of abdominal pregnancy is difficult as patient commonly presents with acute abdomen and shock.

\section{CASE REPORT}

22-year-old female gravida 1 , abortion 1 , belonging to low socio-economic class presented to the emergency department at our hospital with 6 weeks amenorrhoea and abdominal pain. Urine beta hCG test was positive and patient had not undergone any prior obstetric ultrasonography. Physical examination revealed mild tachycardia, BP 90/60 $\mathrm{mm}$ of $\mathrm{Hg}$ and abdominal guarding and rigidity. USG showed a well-defined hypoechoic cystic lesion in the spleen with small heterogenous hyperechoic dependent component within. Free fluid was seen in the pouch of Douglas. Uterus and both adnexa appeared normal. Patient underwent emergency laparotomy with a differential diagnosis of ?splenic hemangioma ?ruptured ectopic pregnancy. Intraoperatively cystic lesion was noted in the spleen. Uterus and adnexa appeared normal. Patient underwent splenectomy.

Gross examination: Specimen of total splenectomy was received at the department of pathology measuring 
$9 \times 5 \times 3 \mathrm{~cm}$ and weight $200 \mathrm{gm}$. Lower pole showed cystic lesion covered by capsule measuring $3 \times 2 \times 2 \mathrm{~cm}$. Spleen was deeply congested, soft to firm. No evidence of any laceration was noted (Figure 1). Sections from cystic lesion and splenic tissue were submitted and studied histopathologically.
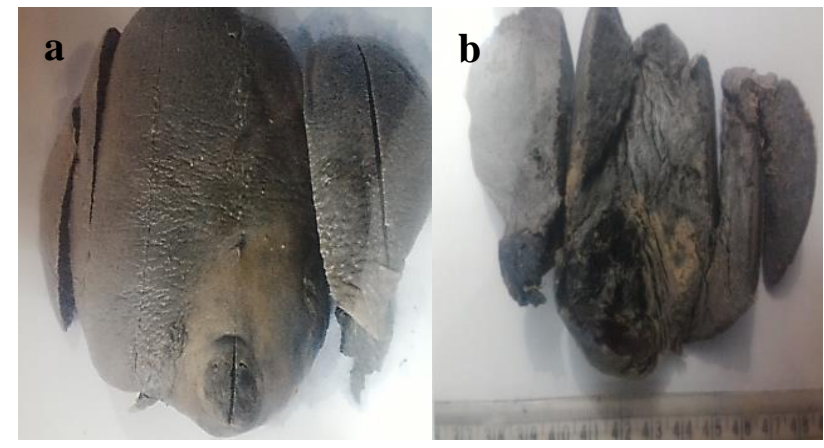

Figure 1: Cystic sac like lesion at lower side of spleen.

Light microscopy: Hematoxylin and eosin stained sections showed presence of chorionic villi with mesenchymal core and lining of cytotrophoblasts and syncytiotrophoblasts invading the splenic parenchyma. Surrounding spleen showed red pulp congestion. (Figure 2). Diagnosis of splenic gestation was given.

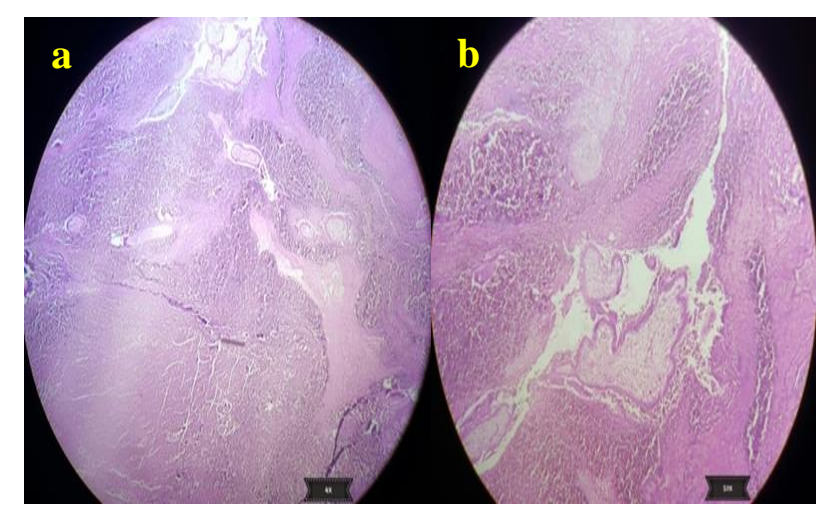

Figure 2: Gestational trophoblastic tissue composed of chorionic villi lined by cytotrophoblast and syncytiotrophoblast in the splenic parenchyma.

\section{DISCUSSION}

Ectopic pregnancy is a leading cause of maternal death in the first trimester of pregnancy. ${ }^{5}$ The estimated incidence of ectopic pregnancies is approximately 19.7 cases per 1000 pregnancies. $^{2}$ The hallmark of ectopic pregnancy is abdominal pain with spotting usually occurring 6 to 8 weeks after the last menstrual period. ${ }^{6}$ Abdominal pregnancy implies pregnancy within the peritoneal cavity and excludes tubal, ovarian and intra-ligamentous pregnancies. $^{7}$ Abdominal pregnancies are classified as primary or secondary. Secondary abdominal pregnancies are by far the most common and result from tubal abortion or rupture or, less often from subsequent implantation within the abdomen after uterine rupture. ${ }^{1}$
Primary abdominal pregnancies resulting from fertilisation of ovum within the peritoneal cavity are rare. ${ }^{7}$ The diagnosis of primary abdominal pregnancy rests on the Studdiford's criteria which includes (a) normal tubes and ovaries with no evidence of recent or past pregnancy (b) no evidence of uteroplacental fistula and (c) the presence of a pregnancy related exclusively to the peritoneal surface and early enough to eliminate the possibility of secondary implantation following primary tubal nidation. ${ }^{8}$ In present case, fallopian tubes and ovaries were normal on USG and also intra operatively. There was no evidence of any uteroplacental fistula and it was an early pregnancy of 6 weeks. Hence a primary splenic pregnancy was confirmed. In our case, no foetal parts were identified grossly or microscopically. The pathogenesis of primary abdominal pregnancies remains an enigma. The pathogenesis has several components including extra tubal fertilisation, transport mechanism and peritoneal implantation. ${ }^{9}$ Extra tubal fertilisation is possible in cases of primary ovarian pregnancy and with IVF techniques. ${ }^{9}$ Cavanagh proposed the postmature ovum as the one where intra tubal fertilisation takes place but reflux occurs resulting in primary ovarian or peritoneal pregnancy. ${ }^{10}$ However it is more likely that the ovum matures early, becomes fertilised within the abdominal cavity, thereafter failing to achieve entry through the tubal ostium.

Normally there is a close anatomical association between the fimbria and the ovulating follicle. Additionally, the presence and quality of cilia, the smooth muscle activity and differential blood flow in the fimbrial folds, all may be distorted by physical or hormonal changes caused by prior salpingitis even though the damage histologically may not be severe. ${ }^{10}$ The other identified risk factors for ectopic pregnancy include very young or old age, previous ectopic pregnancy, previous pelvic surgery, tubal pathology, tubal sterilisation, history of pelvic inflammatory disease, use of IUD, smoking, infertility, IVF and other assisted reproductive techniques. In our case however, none of the risk factors were identified.

The spleen may be relatively more favourable for implantation considering the fact that it is a flat organ rich in blood supply and easily reached in the human supine position by the fertilised ovum. ${ }^{11}$ However it cannot accommodate entire growing embryo and hence there is a risk of rupture and massive intra peritoneal hemorrhage. Spleen is a very rare site for implantation and so a clinical diagnosis was difficult. Because conservative management was thought to be inappropriate and with the clinical diagnostic dilemma this patient underwent exploratory laparotomy and total splenectomy was done.

\section{CONCLUSION}

High index of suspicion is required to diagnose a case of splenic gestation and avoid the consequent potential morbidity and mortality. 
USG is the usual diagnostic procedure of choice but findings are dependent on the examiners experience and quality of ultrasound.

Although CT scan and MRI are not commonly used in imaging of patients with positive beta hCG test due to known hazards, various types of ectopic pregnancies can be confirmed with these modalities. We conclude splenectomy as the current definitive management of rare cases of primary splenic pregnancy.

\section{ACKNOWLEDGMENTS}

Authors would like to thank Dean Dr. Shinde A.D. and technical staff of Department of Pathology, GMC, Latur.

Funding: No funding sources

Conflict of interest: None declared

Ethical approval: Not required

\section{REFERENCES}

1. Thomas G. Stovall. Early Pregnancy Loss and Ectopic Pregnancy. Novak's Gynecology. 13th ed. Lippincott Williams and Wilkins. 2002:507-532.

2. Centers for Disease Control and Prevention. Ectopic pregnancy: United States, 1990-1992. MMWR Morb Mortal Wkly Rep. 1995;44:46-8.
3. Bouyer J, Coste J, Fernandez H, Pouly JL, Job-Spira N. Sites of ectopic pregnancy: a 10 year population based study of 1800 cases. Hum Reprod. 2002; 17:3224-30.

4. Siddiqui NA, Islam T. Abdominal Pregnancy Implanted in the Spleen A case report; AKMMC J 2011;2(2):36-8.

5. Farquhar CM. Ectopic pregnancy. Lancet. 2005;366 (9485):583-91.

6. Tenore JL. Ectopic pregnancy. Am Fam Physician 2000:61:1080-8.

7. Delke I, Veridiano NP, Tancer ML. Abdominal pregnancy: review of current management and addition of 10 cases. Obstet Gynecol. 1982;60(2):200-4.

8. Studdiford WE: Primary peritoneal pregnancy. Am J Obstet Gynecol. 1942;44:487.

9. Thomas JS, Willie JO. Primary peritoneal pregnancy: a case report. J Natl Med Assoc. 1991;83:635-7.

10. Cavanagh D. Primary peritoneal pregnancy. Am J Obstet Gynecol. 1966;96:511-20.

11. Yagil Y, Beck-Razi N. Splenic Pregnancy The role of abdominal imaging. J Ultrasound Med. 2007;26:1629-32.

Cite this article as: Patil NR, Birare SD, Dale AR, Mahule SK. Splenic Gestation: A rare case report. Int J Reprod Contracept Obstet Gynecol 2017;6:2670-2. 